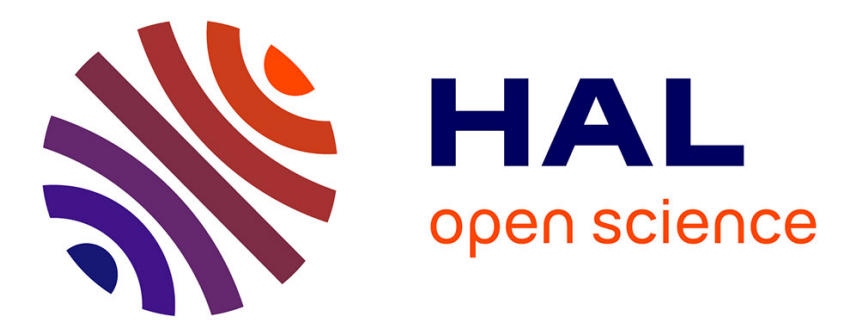

\title{
Kinetic over-relaxation method for the convection equation with Fourier solver
}

Romane Hélie, Philippe Helluy, Emmanuel Franck, Laurent Navoret

\section{To cite this version:}

Romane Hélie, Philippe Helluy, Emmanuel Franck, Laurent Navoret. Kinetic over-relaxation method for the convection equation with Fourier solver. Finite Volumes for Complex Applications IX - Methods, Theoretical Aspects, Examples. FVCA 2020., pp.745-753, 2020, 978-3-030-43650-6. 10.1007/9783-030-43651-3_71. hal-02427044v2

\section{HAL Id: hal-02427044 \\ https://hal.science/hal-02427044v2}

Submitted on 23 Mar 2021

HAL is a multi-disciplinary open access archive for the deposit and dissemination of scientific research documents, whether they are published or not. The documents may come from teaching and research institutions in France or abroad, or from public or private research centers.
L'archive ouverte pluridisciplinaire HAL, est destinée au dépôt et à la diffusion de documents scientifiques de niveau recherche, publiés ou non, émanant des établissements d'enseignement et de recherche français ou étrangers, des laboratoires publics ou privés. 


\title{
Kinetic over-relaxation method for the convection equation with Fourier solver
}

\author{
Romane Hélie ${ }^{1,2}$, Philippe Helluy ${ }^{1,2}$, \\ Emmanuel Franck ${ }^{1,2}$, Laurent Navoret ${ }^{1,2}$
}

1. Institut de Recherche Mathématique Avancée, UMR 7501, Université de Strasbourg et CNRS, 7 rue René Descartes, 67000 Strasbourg, France

2. INRIA Nancy-Grand Est, TONUS Project, Strasbourg, France

\begin{abstract}
In this paper, we apply the CFL-less kinetic over-relaxation scheme presented in [1] to the convection equation in two space dimensions. The method is a succession of free-transport steps and collisions steps. The free transport steps are solved with Fourier discretization. The collision steps are solved with over-relaxation for achieving high order. The method reaches six-order accuracy when using palindromic composition method. We apply the method to the guiding-center model in plasma physics.
\end{abstract}

keywords: relaxation, composition, Fourier, guiding center model

MSC (2010): 35L65, 65M12, 65T50, 35Q83, 82D10

\section{Introduction}

The kinetic over-relaxation method [1] is a time semi-discrete method based on the approximation of a non-linear convection equation by a set of linear transport equations with constant velocities. Very efficient, CFL-less, and accurate transport solvers like Fourier methods can be used. Moreover, the over-relaxation technic lead to second-order accuracy in time. Even higher order can be achieved by composition methods. In this paper, we apply these methods to the convection equation in two-dimension and we show that it is particularly appropriate to solve the guiding center model, where the convection velocity field is given by a solution to a Poisson equation. The guiding center model is a simplified model to describe the twodimensional dynamics of the charge density in a Tokamak. The particles are confined in the toroidal room thanks to a large external magnetic field $B$. Among several 
dynamics, this magnetic field leads to the so-called $E \times B$ drift of the particles, where $E$ is the self-induced electric field. This model is also equivalent to the $2 \mathrm{~d}$ incompressible Euler equation in the vorticity formulation. The dynamics result in very fine scale structures and thus require very accurate solvers.

\section{Kinetic over-relaxation approximation of the con- vection equation}

We consider the following convection equation:

$$
\partial_{t} \rho(t, \boldsymbol{x})+\nabla \cdot(\rho(t, \boldsymbol{x}) \boldsymbol{a}(t, \boldsymbol{x}))=0,
$$

where $\boldsymbol{a}(t, \boldsymbol{x}) \in \mathbb{R}^{d}$ is the velocity field and $\rho(t, \boldsymbol{x}) \in \mathbb{R}$ is the convected density.

To solve this convection equation with non-constant velocity field, the relaxation method consists in approximating it with several transport equations at constant velocities. More precisely, we introduce a kinetic vector $\boldsymbol{f}(t, \boldsymbol{x})=\left(f_{1}(t, \boldsymbol{x}), f_{2}(t, \boldsymbol{x}), \ldots\right.$, $\left.f_{N}(t, \boldsymbol{x})\right) \in \mathbb{R}^{N}$, whose components are associated to different velocities $\left(\boldsymbol{\lambda}_{1}, \ldots, \boldsymbol{\lambda}_{N}\right) \in$ $\left(\mathbb{R}^{d}\right)^{N}$. To a given kinetic vector $\boldsymbol{f}(t, \boldsymbol{x})$, we associate a macroscopic density

$$
\rho_{\boldsymbol{f}}(t, \boldsymbol{x})=\sum_{i=1}^{N} f_{i}(t, \boldsymbol{x}) .
$$

The numerical scheme is devised such that $\rho_{\boldsymbol{f}}$ is an approximation of the solution $\rho$. To this end, for any given density $\rho \in \mathbb{R}$, we introduce the so-called equilibrium kinetic vector $\boldsymbol{f}_{[\boldsymbol{a}, \rho]}^{\mathrm{eq}}$ that satisfies the following consistency relations:

$$
\rho=\sum_{i=1}^{N} f_{[\boldsymbol{a}, \rho], i}^{\mathrm{eq}}, \quad \rho \boldsymbol{a}=\sum_{i=1}^{N} \boldsymbol{\lambda}_{i} f_{[\boldsymbol{a}, \rho], i}^{\mathrm{eq}} .
$$

The scheme is based on a time discretization of the following equation:

$$
\partial_{t} \boldsymbol{f}+\sum_{k=1}^{d} \boldsymbol{\Lambda}_{k} \partial_{x_{k}} \boldsymbol{f}=\frac{1}{\varepsilon}\left(\boldsymbol{f}_{\left[\boldsymbol{a}, \rho_{\boldsymbol{f}}\right]}^{\mathrm{eq}}-\boldsymbol{f}\right),
$$

where $\boldsymbol{\Lambda}_{k}=\operatorname{diag}\left(\left(\boldsymbol{\lambda}_{1}\right)_{k}, \ldots,\left(\boldsymbol{\lambda}_{2}\right)_{k}\right)$ are $N \times N$ diagonal matrices, for $k=1, \ldots, d$, and where $\varepsilon>0$ is a small parameter that controls the distance to the equilibria set. In the time-discretization, the time-dependent relaxation operator in the r.h.s. is replaced by a projection onto the equilibria set or a symmetry with respect to the equilibria set or a combination of the two.

The time semi-discretization of the over-relaxation scheme writes as follows. We start from the equilibrium distribution associated with the initial data: $\boldsymbol{f}(0, \boldsymbol{x})=$ $\boldsymbol{f}_{\left[\boldsymbol{a}(0, \boldsymbol{x}), \rho_{0}(\boldsymbol{x})\right]}^{\text {eq }}$. Then, at each time step $\Delta t>0$, starting from $\boldsymbol{f}(t, \boldsymbol{x})$, we compute $\boldsymbol{f}(t+\Delta t, \boldsymbol{x})$ in two steps:

1. (transport step) advect the several kinetic components $f_{i}$ with their respective velocities $\boldsymbol{\lambda}_{i} \in \mathbb{R}^{d}$

$$
f_{i}^{*}(t+\Delta t, \boldsymbol{x})=f_{i}\left(t, \boldsymbol{x}-\Delta t \boldsymbol{\lambda}_{i}\right), \quad \forall i \in\{1, \ldots, N\},
$$

which is also denoted in compact form: $\boldsymbol{f}^{*}(t+\Delta t,)=.T(\Delta t) \boldsymbol{f}(t,$.$) .$ 
2. (over-relaxation step) compute $\rho_{\boldsymbol{f}^{*}(t+\Delta t, .)}$ and then perform the following relaxation

$$
\boldsymbol{f}(t+\Delta t, .)=\boldsymbol{f}^{*}(t+\Delta t, \boldsymbol{x})+\omega\left(\boldsymbol{f}_{\left[\boldsymbol{a}(t+\Delta t, .), \rho_{\boldsymbol{f}^{*}}(t+\Delta t, .)\right]}^{\mathrm{eq}}-\boldsymbol{f}^{*}(t+\Delta t, \boldsymbol{x})\right),
$$

with $\omega \in[1,2]$ a given parameter, also denoted: $\boldsymbol{f}(t+\Delta t,)=.R_{\omega} \boldsymbol{f}^{*}(t+\Delta t,$.$) .$ For $\omega=1$, we obtain the projection onto the equilibria set and for $\omega=2$, we get the symmetry w.r.t the equilibria set.

The combination of these two steps writes as follows:

$$
\boldsymbol{f}(t+\Delta t, .)=M_{1}(\Delta t) \boldsymbol{f}(t, .), \quad \text { with } M_{1}(\Delta t)=\left(R_{\omega} \circ T(\Delta t)\right)
$$

Then $\rho_{\boldsymbol{f}}$ is a first-order approximation of the solution $\rho$ to (1) for $\omega<2$ and a second-order approximation if $\omega=2$. We refer the reader to [1,2] as regards the corresponding equivalent equation. From this equivalent equation, we can infer the so-called sub-characteristic condition that ensures the dissipativity of the secondorder term in the expansion.

As presented in [1], higher-order time discretization can be devised by considering the following second-order time-symmetric operator:

$$
M_{2}(\Delta t)=\left(T\left(\frac{\Delta t}{4}\right) \circ R_{2} \circ T\left(\frac{\Delta t}{2}\right) \circ R_{2} \circ T\left(\frac{\Delta t}{4}\right)\right),
$$

and then using a palindromic composition method

$$
M_{p}(\Delta t)=M_{2}\left(s_{0} \Delta t\right) \circ M_{2}\left(s_{1} \Delta t\right) \circ \cdots \circ M_{2}\left(s_{p} \Delta t\right),
$$

where $s_{i}=s_{p-i}$, for $i=0, \ldots, p$. We will consider the fourth-order Suzuki scheme $(p=4)$ and the sixth order Kahan-Li scheme $(p=8)$. We refer to [1] for the expression of the corresponding parameters.

This numerical scheme has the advantage to concentrate all the non-linear operators in a local step, while the transport step becomes fully linear. Therefore, CFLless method can be employed to make these transport steps. A semi-Lagrangian scheme has been used in [2]. On non-Cartesian meshes, implicit Discontinuous Galerkin method with upwind fluxes can be used as proposed in [1]. Here, we consider a Fourier discretization of the transport equation, ensuring a spectral accuracy.

In the sequel, we will use the so-called [D2Q4] kinetic approximation $(N=4)$. It consists in introducing the four velocities directed along the Cartesian axes:

$$
\lambda_{1}=\left[\begin{array}{c}
\lambda \\
0
\end{array}\right], \quad \lambda_{2}=\left[\begin{array}{c}
0 \\
\lambda
\end{array}\right], \quad \lambda_{3}=\left[\begin{array}{c}
-\lambda \\
0
\end{array}\right], \quad \lambda_{4}=\left[\begin{array}{c}
0 \\
-\lambda
\end{array}\right]
$$

with $\lambda>0$ and then we define the kinetic equilibrium vector:

$$
f_{[\boldsymbol{a}, \rho], i}^{\mathrm{eq}}=\frac{\rho}{4}+\frac{\rho\left(\boldsymbol{a} \cdot \boldsymbol{\lambda}_{i}\right)}{2 \lambda^{2}}, \quad \forall i \in\{1,2,3,4\} .
$$

This is the only solution to consistency relations (2), which satisfies symmetries. The sub-characteristic condition writes in that case: $\lambda>\max _{[0, T] \times \Omega}\|\boldsymbol{a}(t, \boldsymbol{x})\|$, where $[0, T] \times \Omega$ is the computational domain. 
We will also consider the [D2Q5] kinetic approximation $(N=5)$, where a fifth central null velocity is added:

$$
\lambda_{1}=\left[\begin{array}{l}
\lambda \\
0
\end{array}\right], \quad \lambda_{2}=\left[\begin{array}{l}
0 \\
\lambda
\end{array}\right], \quad \lambda_{3}=\left[\begin{array}{c}
-\lambda \\
0
\end{array}\right], \quad \lambda_{4}=\left[\begin{array}{c}
0 \\
-\lambda
\end{array}\right], \quad \lambda_{5}=\left[\begin{array}{l}
0 \\
0
\end{array}\right] .
$$

where $\lambda>0$. The kinetic equilibrium vector has to satisfy consistency relations (2):

$$
\begin{aligned}
\rho & =f_{[\boldsymbol{a}, \rho], 1}^{\mathrm{eq}}+f_{[\boldsymbol{a}, \rho], 2}^{\mathrm{eq}}+f_{[\boldsymbol{a}, \rho], 3}^{\mathrm{eq}}+f_{[\boldsymbol{a}, \rho], 4}^{\mathrm{eq}}+f_{[\boldsymbol{a}, \rho], 5}^{\mathrm{eq}}, \\
\rho a_{1} & =\lambda\left(f_{[\boldsymbol{a}, \rho], 1}^{\mathrm{eq}}-f_{[\boldsymbol{a}, \rho], 3}^{e q}\right), \quad \rho a_{2}=\lambda\left(f_{[\boldsymbol{a}, \rho], 2}^{\mathrm{eq}}-f_{[\boldsymbol{a}, \rho], 4}^{e q}\right) .
\end{aligned}
$$

This system is underdetermined. As already proposed in [2] for the one-dimensional case, we consider the following decomposition based on a flux-splitting

$$
f_{[\boldsymbol{a}, \rho], i}^{\mathrm{eq}}=\rho\left(\boldsymbol{\lambda}_{i} \cdot \boldsymbol{a}\right)_{+}, \quad \forall i \in\{1,2,3,4\}, \quad f_{[\boldsymbol{a}, \rho], 5}^{\mathrm{eq}}=\rho-\sum_{i=1}^{4} f_{[\boldsymbol{a}, \rho], i}^{\mathrm{eq}},
$$

where for any $v \in \mathbb{R}, v_{+}=\max \{v, 0\}$ stands for the positive part of $v$ or can be approximated by a smooth version $v_{+}=\left(v+H_{r}(v)\right) / 2$ where $H_{r}(v)$ are Halley's functions defined recursively by: $H_{0}(x)=1, H_{r+1}(x)=H_{r}(x)\left(H_{r}(x)^{2}+\right.$ $\left.3 x^{2}\right) /\left(3 H_{r}(x)^{2}+x^{2}\right)$. The sub-characteristic condition is the same as for the [D2Q4] approximation. As explained in [2], this scheme is expected to be more precise and better captures unidirectional flows.

\section{Numerical results}

In this section, we validate the numerical scheme on two test-cases : the rotation advection test-case and the Kelvin-Helmholtz test-case for the guiding-center model. In these two test-cases, the transport part $T(\Delta t)$ is discretized with a Fourier method.

Rotation test-case We consider the convection equation (1) where the velocity field is given by $\boldsymbol{a}(\boldsymbol{x})=\boldsymbol{x}^{\perp}$. This velocity field is divergence free: $\nabla \cdot \boldsymbol{a}=0$. Therefore, the convection equation (1) is equivalent to the advection equation:

$$
\partial_{t} \rho(t, \boldsymbol{x})+\boldsymbol{a}(\boldsymbol{x}) \cdot \nabla \rho(t, \boldsymbol{x})=0,
$$

and the exact solution is just the rotation of the initial density around the origin.

In the following, we consider the domain $\Omega=[-1,1] \times[-1,1]$ and the exact solution:

$$
\rho(t, \boldsymbol{x})=\frac{1}{2 \pi} \exp \left(-\frac{\left\|R(t)\left(\boldsymbol{x}-\boldsymbol{x}_{0}\right)\right\|^{2}}{\sigma^{2}}\right) .
$$

where $\sigma=0.1$ and $\boldsymbol{x}_{0}=(0.5,0)$ and $R(t)$ is the rotation matrix of angle $t$. We use $N_{x}=N_{y}=200$ discretization points in each direction.

Figure 1 (left) shows that the [D2Q4] scheme is first order accurate with the relaxation parameter $\omega=1.95$. Second order accuracy is achieved with $\omega=2$ for both [D2Q4] and [D2Q5] using the $M_{1}$ operator. As expected, we also note that the [D2Q5] is more accurate than the [D2Q4] scheme.

In Figure 1 (right), we observe that the $M_{2}$ operator is required to obtain the sixth-order accuracy of the Kahan-Li composition method. Using the $M_{1}$ 

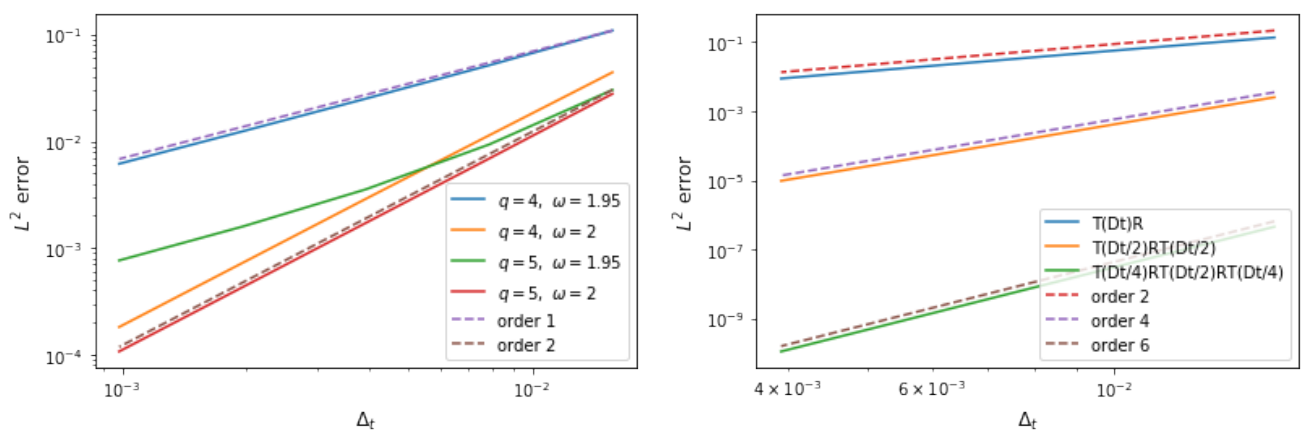

Figure 1: $L^{2}$ error between the exact and the numerical solution obtained as function of the time step. Left: Comparison between $q=4$ ([D2Q4]) and $q=5([D 2 Q 5], r=4)$ for different $\omega$. Right: Comparison between different splitting operators when using the $[D 2 Q 5]$ method $(r=4)$ with a Kahan-Li palindromic composition and $\omega=2$. Parameters: $\lambda=2.1, N_{x}=N_{y}=200$.

Table 1: Number of time steps and execution times needed to achieve an accuracy of $10^{-8}$ at time $T=\pi / 2$ with $\lambda=2.1, N_{x}=N_{y}=200$.

$\begin{array}{cccc}\text { Nb of } & \text { Nb of } & \text { Error } L^{2} & \text { Exec. time } \\ \text { time steps } & \text { transport steps } & & \end{array}$

\begin{tabular}{lcccc}
{$[D 2 Q 5], \omega=2, M_{1}$} & 172000 & 172000 & $9.258 \times 10^{-9}$ & 4985.282 \\
\hline$[D 2 Q 5], \omega=2, M_{2}$ & 82000 & 246000 & $9.975 \times 10^{-9}$ & 6298.363 \\
\hline$[D 2 Q 5], \omega=2, M_{2}$, Suzuki & 570 & 8550 & $9.516 \times 10^{-9}$ & 223.407 \\
\hline$[D 2 Q 5], \omega=2, M_{2}$, Kahan-Li & 190 & 5130 & $9.924 \times 10^{-9}$ & 145.426 \\
\hline$[D 2 Q 4], \omega=2, M_{2}$, Kahan-Li & 215 & 5805 & $9.627 \times 10^{-9}$ & 132.539 \\
\hline
\end{tabular}

operator leads to a second order operator and the Strang splitting $M_{2}^{S}(\Delta t)=$ $\left(T\left(\frac{\Delta t}{2}\right) \circ R_{2} \circ T\left(\frac{\Delta t}{2}\right)\right)$ to a fourth-order accuracy only.

As regards the computational time, we observe in Table 1 that considering $M_{1}$ is 1.26 times more efficient than considering $M_{2}$ when $\omega=2$. Indeed, both methods are of order 2 and $M_{1}$ requires less transport steps. However, using $M_{2}$, we can use the Suzuki or the Kahan-Li composition methods that are respectively 22 and 34 times faster. For these comparisons, we use the [D2Q5] method. The [D2Q4] method seems just as fast even though it requires more transport steps. Although more accurate, the [D2Q5] is slowed down by the evaluation of the Halley functions.

Kelvin-Helmholtz test-case We consider the guiding center model that describes the two-dimensional dynamics of electrons resulting from the $E \times B$ drift due to a large magnetic field. Their charge density is denoted $\rho(t, x)>0$ and the guiding center model writes :

$$
\begin{aligned}
& \partial_{t} \rho+E^{\perp} \cdot \nabla \rho=0, \\
& -\Delta \phi=\rho_{0}-\rho, \quad E=-\nabla \phi .
\end{aligned}
$$


where $E(t, x) \in \mathbb{R}^{d}$ the electric field and $\phi(t, x) \in \mathbb{R}$ the electric potential. $\rho_{0}(t)>0$ denotes the ion background charge density, which is supposed homogeneous. Actually, this model is equivalent to the $2 \mathrm{~d}$ incompressible Euler equation in the vorticity formulation. Here we consider a square domain $\Omega$ with periodic boundary conditions and we thus assume that $\rho_{0}(t)$ equals the average of the density over the domain: $\rho_{0}(t)=\frac{1}{|\Omega|} \int_{\Omega} \rho(t, x) d x$.

Since $E=-\nabla \phi$, the advection vector field $E^{\perp}$ is divergence free. The transport equation is thus equivalent to the conservative convection equation

$$
\partial_{t} \rho+\nabla \cdot\left(\rho E^{\perp}\right)=0,
$$

Unlike the previous advection equations presented so far, here the advection field depends on the density itself. Therefore, the over-relaxation scheme is slightly modified and writes:

1. (transport step) $f^{*}(t+\Delta t,)=.T(\Delta t) \boldsymbol{f}(t,$.$) ,$

2. (Poisson step) compute $\left.\rho_{\boldsymbol{f}^{*}(t+\Delta t},.\right)$ and then find $\phi^{*}(t+\Delta t,$.$) by solving the$ Poisson equation and then $\boldsymbol{a}(t+\Delta t,)=.E^{*}(t+\Delta t, .)^{\perp}$.

3. (over-relaxation step) $f(t+\Delta t,)=.R_{\omega} f^{*}(t+\Delta t,$.$) .$

Note that both the transport step and the Poisson equation can be solved using a Fourier discretization in the square domain.

As already considered in [3-5], the Kelvin-Helmholtz instability test-case consists in considering the following initial condition:

$$
\rho_{\text {init }}(x, y)=\sin x+\varepsilon \cos (k y),
$$

in the domain $[0,2 \pi] \times[0,2 \pi / k]$, with periodic boundary conditions, and where $k \in \mathbb{R}$ is the perturbation wave number and $\varepsilon>0$ is the perturbation amplitude. This is a perturbation of the stationary solution $\rho_{0}(x)=\sin x$ and $\phi_{0}(x)=-\sin x$. According to [4], there exists a critical wave number $k_{s}=1$ such that an instability develops only for $k<k_{s}$. The instability rates are not known explicitly. However, we can compute them numerically.

We look for solutions of the form

$$
\rho(x, y, t)=\rho_{0}(x)+\varepsilon \rho_{1}(x, y, t), \quad \phi_{0}(x, t)=\phi_{0}(x)+\varepsilon \phi_{1}(x, y, t)
$$

where $\rho_{1}(x, y, t)=\tilde{\rho}_{1}(x) \exp (i k y) \exp (-i \omega t), \phi_{1}(x, y, t)=\tilde{\phi}_{1}(x) \exp (i k y) \exp (-i \omega t)$. Following [4], it can be proved that $\tilde{\phi}_{1}$ solves the generalized eigenvalue problem:

$$
\phi_{0}^{\prime}\left(\partial_{x^{2}} \tilde{\phi}_{1}-k^{2} \tilde{\phi}_{1}\right)+\tilde{\phi}_{1} \rho_{0}^{\prime}=-\omega / k \phi_{0}^{\prime}\left(\partial_{x^{2}} \tilde{\phi}_{1}-k^{2} \tilde{\phi}_{1}\right),
$$

in which $\omega / k$ stands for the eigenvalue. As explained in [4], it can be proved that unstable solutions, corresponding to $\omega / k$ with positive imaginary part, exist if and only if $k<k_{s}=1$. For $k$ near $k_{s}$, a first order approximation of the instability rate can be computed: $\omega / k=2\left(k_{s}-k\right) i$. Alternatively, we can also compute the instability rate by solving (6) numerically using a finite difference method. Introducing a space step $\Delta x=1 / N$ with $N \in \mathbb{N}$ and the corresponding spatial discretization of the interval $[0,1], x_{i}=i \Delta x$, we consider the approximate solution $\Phi_{1} \in \mathbb{C}^{N}$, such that $\left(\Phi_{1}\right)_{i} \approx \tilde{\phi}_{1}\left(x_{i}\right)$ and which solves the following problem

$$
C\left(D+\left(1-k^{2}\right) \mathrm{Id}\right) \Phi_{1}=\omega / k\left(D-k^{2} \mathrm{Id}\right) \Phi_{1},
$$



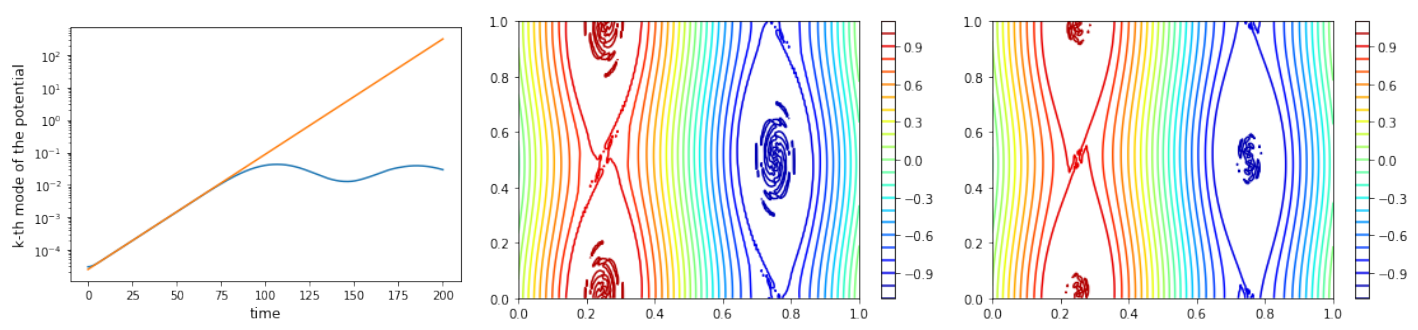

Figure 2: (Kelvin-Helmholtz, $k=0.95, \varepsilon=10^{-4}, N_{x}=N_{y}=200, \Delta t=0.01$, [D2Q5], $r=4, \lambda=2.02$ ) Left: Time evolution of the $k$-th Fourier mode of the potential (in blue) and the straight line with slope $\operatorname{Im}(\omega)=0.08185$ (in orange) with Kahan-Li, $\omega=2$. Middle and left: Contour lines of the density at final time $T=200$ with $\omega=2$, Kahan-Li (middle) and $\omega=1.95, M_{2}$ (right).

where $C=\operatorname{diag}\left(\cos \left(x_{1}\right), \ldots, \cos \left(x_{N}\right)\right)$ is diagonal matrix and $D$ is discrete Laplacian matrix with periodic boundary conditions. Therefore, assembling $A=C(D+$ $\left.\left(1-k^{2}\right) \mathrm{Id}\right)$ and $B=\left(D-k^{2} \mathrm{Id}\right)$, we just have to compute numerically the eigenvalues of the matrix $B^{-1} A$ and then keep the one with the largest imaginary part.

In Figure 2 (left) is plotted the time evolution of the $k$-th Fourier mode of the potential. The instability rate fits perfectly with the expected one obtained solving (7). In the middle and right are plotted the contour lines of the density with the first-order scheme $M_{1}$ and the Kahan-Li composition methods. This illustrates the need to use high order scheme to capture the small structures.

\section{Conclusion}

In this paper, we show that the kinetic over-relaxation method enables to devise numerical schemes for the convection equation based on Fourier discretization. The proposed method is optimally high-order accurate in space and can reach sixth order time accuracy with the Kahan-Li composition method. Unless high order schemes require more intermediate transport steps, the computational cost can be drastically decreased. Moreover, the method has been extended to the non-linear guiding center model. This is the first step before the extension to more complex advection equations like the gyro-kinetic equation in plasma physics.

Acknowledgement This work has been carried out within the framework of the EUROfusion Consortium and has received funding from the Euratom research and training programme 2014-2018 and 2019-2020 under grant agreement No 633053. The views and opinions expressed herein do not necessarily reflect those of the European Commission

\section{References}

[1] D. Coulette, E. Franck, P. Helluy, M. Mehrenberger, and L. Navoret. Highorder implicit palindromic discontinuous Galerkin method for kinetic-relaxation 
approximation. Comput. Fluids, 190:485-502, 2019.

[2] C. Courtès, D. Coulette, E. Franck, and L. Navoret. Vectorial kinetic relaxation model with central velocity. application to implicit relaxations schemes. Commun. Comput. Phys., 2018.

[3] N. Crouseilles, M. Mehrenberger, and E. Sonnendrücker. Conservative semiLagrangian schemes for Vlasov equations. J. Comput. Phys., 229(6):1927-1953, 2010.

[4] M. M. Shoucri. A two-level implicit scheme for the numerical solution of the linearized vorticity equation. Int. J. Numer. Meth. Eng., 17(10):1525-1538, 1981.

[5] E. Sonnendrücker, J. Roche, P. Bertrand, and A. Ghizzo. The semi-Lagrangian method for the numerical resolution of the Vlasov equation. J. Comput. Phys., 149(2):201-220, 1999. 